\title{
Fermentation Monitoring of a Co-Culture Process with Saccharomyces cerevisiae and Scheffersomyces stipitis Using Shotgun Proteomics
}

\section{Eric L Huang and Mark G Lefsrud}

Bioresource Engineering, McGill University, 21111 Lakeshore Road, Ste-Anne-de-Bellevue, Quebec, H9X 3V9, Canada

\begin{abstract}
Co-culture processes present the opportunity to produce value-added products from economical raw materials, but there lacks a high-throughput fermentation monitoring technique to study the temporal physiology of fermentation organisms in co-culture processes. In this study, we applied shotgun proteomics to investigate a co-culture process of Saccharomyces cerevisiae and Scheffersomyces stipitis, and we monitored the fermentation until glucose depletion. Three time points were taken for proteomics analysis at 11.5 hour, 18.5 hour and 32 hour, representing transition into diauxic shift. Using label-free quantitation, we observed cellular dynamic within 20-hour time frame. We distinguished the proteome between two yeasts, and the most abundant proteins of $S$. stipitis and $S$. cerevisiae contained expected processes of glycolytic enzymes, histones, heat shock proteins, ribosomal proteins and $F_{1} F_{0}$-ATPase. After glucose depletion, we identified up-regulations of $S$. stipitis malate synthase and isocitrate lyase as key enzymes in glyoxylate cycle and gluconeogenesis. Increased expression of S. stipitis histone 2B was observed in diauxic shift, and histone acetylation was suggested by up-regulation of acetyl-CoA synthetase. Without the presence of xylose, we observed induction of $\mathrm{NAD}(\mathrm{P}) \mathrm{H}$-dependent $\mathrm{D}$-xylose reductase $(\mathrm{Xyl} / \mathrm{p})$ as early as 11.5-hour before glucose depletion. We also observed the expression of D-xylulose reductase after glucose depletion without xylose induction. Further study is needed to investigate the cause of derepression signals for xylose oxo-reductive pathway. Without cellulose induction, the up-regulation of S. stipitis endo-1,4-beta-glucanase suggested S. stipites' strategy in diversifying carbon choices after glucose repression. This research demonstrated the application of shotgun proteomics in high-throughput monitoring of complex co-culture system and able to elucidate the temporal physiology of $S$. stipitis.
\end{abstract}

Keywords: Saccharomyces cerevisiae; Scheffersomyces stipitis; Pichia stipitis; Shotgun proteomics; Co-Culture; Mudpit; Fermentation monitoring

\section{Introduction}

Co-culture processes present the opportunity to establish stable and profitable biotechnological bioprocesses and produce valueadded products from economical raw materials such as agricultural residues [1]. Mixed culture systems have demonstrated promise in hydrogen, methane, ethanol and polyhydroxyalkanoates productions from renewable resource, increasing potential revenue and reducing environmental impacts [2-4]. The use of controlled mixed fermentation using Saccharomyces and non-Saccharomyces yeasts has been implemented in winemaking to alter both chemical and the aromatic composition of wines [5]. The synergistic interaction between different yeasts provides a tool for the implementation of new fermentation technologies [5]. In biofuel research, the co-culture process of Saccharomyce cerevisiae and Scheffersomyces stipitis (Pichia stipitis) has been extensively studied with attempts to achieve a simple, one-batch process to ferment glucose and xylose using lignocellulosic feedstock [6,7]. The co-culture process poses technical obstacles such as low ethanol yields associated with different optimal oxygen transfer rate required by each organism. Novel fermentation apparatus using two fermentors and two microfiltration modules to achieve optimal fermentation condition for each yeast [7]. However, before optimizing a co-culture process such as ones described by Taniguchi et al. [7], there lacks a robust, high-throughput fermentation monitoring technique to monitor the temporal physiology of each organism in more complex process such as co-culture fermentation. In recent years, fermentation technology has turned '-omics' for solutions in process development [8]. Proteomics quantification of yeast high gravity ethanol fermentations was investigated to determine the dynamic of metabolic pathways (e.g. the deactivation of secondary metabolites pathway) [9]. Shotgun proteomics can provide the physiological backgrounds needed for non- model organisms such as $S$. stipitis and allow observation of the global profile of the organism's proteome. The analysis of the proteome allows the evaluation of cellular processes at greater depth with a much simpler technique than 2D-PAGE-MS [10]. In this study we demonstrated the application of shotgun proteomics in monitoring a co-culture process using S. cerevisiae S288C and S. stipitis CBS 6054. The yeast proteome of three time points were analyzed based on metabolic and regulatory pathways. Without the presence of xylose, we observed the induction of xylose reductase (Xyllp, S. stipitis) as early as 11.5-hour before glucose depletion, and D-xylulose reductase (Xyl2p, S. stipitis) as early as 18.5hour after glucose depletion. . We distinguished the proteome between two yeasts, identified the most abundant proteins of $S$. stipitis and $S$. cerevisiae, and determine the dynamic of $S$. stipitis proteome during the co-culture process.

\section{Materials and Methods}

\section{Microorganism and culture condition}

Saccharomyces cerevisiae S288C and Scheffersomyces stipitis (syn. Pichia stipitis CBS 6054) were obtained from American Type Culture

*Corresponding author: Mark G Lefsrud, Bioresource Engineering, McGill University, 21111 Lakeshore Road, Ste-Anne-de-Bellevue, Quebec, H9X 3V9, Canada, Tel: 514-398-7967; Fax: 514-398-8387; Email: mark.lefsrud@ mcgill.ca

Received October 27, 2013; Accepted January 02, 2014; Published January 09, 2014

Citation: Huang EL, Lefsrud MG (2014) Fermentation Monitoring of a CoCulture Process with Saccharomyces cerevisiae and Scheffersomyces stipitis Using Shotgun Proteomics. J Bioprocess Biotech 4: 144 doi: 10.4172/21559821.1000144

Copyright: (c) 2014 Huang EL, et al. This is an open-access article distributed under the terms of the Creative Commons Attribution License, which permits unrestricted use, distribution, and reproduction in any medium, provided the original author and source are credited. 
Collection (ATCC, Manassas, VA, USA) and maintained on YPD or YPX agar slants accordingly. YPX substituted xylose with glucose in YPD. Pre-inoculums of $S$. cerevisiae and S. stipitis were grown separately at $26^{\circ} \mathrm{C}, 100 \mathrm{rpm}$ and harvested at mid-exponential phase. Two biological replicates of the co-culture process were carried out in a $250 \mathrm{~mL}$ Erlenmeyer flask with $100 \mathrm{~mL}$ working volume at $26^{\circ} \mathrm{C}$ and $100 \mathrm{rpm}$. The medium composition for the co-culture process was $20 \mathrm{~g} / \mathrm{L}$ glucose, $20 \mathrm{~g} / \mathrm{L}$ peptone and $10 \mathrm{~g} / \mathrm{L}$ yeast extract.

\section{Fermentation parameters analysis}

Glucose, ethanol, acetic acid and glycerol were measured using enzymatic kits (Megazyme, Wicklow, Ireland). OD610 was monitored throughout growth with a UV-VIS spectrophotometer. Colonyforming unit (CFU) was used to obtain viable yeast counts of $S$. stipitis and S. cerevisiae. S. stipitis and S. cerevisiae were enumerated by adapting methods as described in Laplace and colleagues [11]. In brief, S. stipitis and S. cerevisiae were distinguished from each other by plating on medium containing erythromycin $(1 \mathrm{~g} / \mathrm{L}), 1 \%$ yeast extract, $2 \%$ peptone, $2 \%$ glycerol or $2 \%$ glucose. S. stipitis was able to grow on media containing erythromycin $(1 \mathrm{~g} / \mathrm{L})$ with $2 \%$ glycerol, whereas $S$. cerevisiae was inhibited by the mitochondrial inhibitor.

\section{Cells lysis and protein extraction}

Three protein samples from each biological replicate were taken at 11.5 hour, 18.5 hour and 32 hour. $\sim 10 \mathrm{mg}$ of wet-mass cell pellet for each sample was obtained and processed through single tube cell lysis and protein digestion $[12,13]$. The protein concentrations after the extraction were $\sim 6 \mathrm{mg} / \mathrm{mL}$ for each sample after extraction. Tris $/ 10 \mathrm{mM}$ $\mathrm{CaCl}_{2}$ at $\mathrm{pH} 7.6$ and $6 \mathrm{M}$ Guanidine/10mM Dithiothreitol (DTT) (Sigma-Aldrich Canada, Oakville, Ontario) were used to lyse cells and extrude proteins. In brief, the mixture was placed on the rocker for the first hour and vortexed every 10 minutes, and then incubated for 12 hours at $37^{\circ} \mathrm{C}$. The mixture was spun down at $10,000 \mathrm{~g}$. The supernatant was discarded and the pellet was diluted with 6 -fold $50 \mathrm{mM}$ Tris $/ 10 \mathrm{mM}$ $\mathrm{CaCl}_{2} . \sim 5 \mu \mathrm{g}$ of sequencing grade trypsin (Promega, Madison, WI, USA) was added to each sample and digested for 12 hours at $37^{\circ} \mathrm{C}$ by gentle rocking. The same amount of trypsin was added a second time and incubated at $37^{\circ} \mathrm{C}$ for another 12 hours. $1 \mathrm{M}$ DTT was added to a final concentration of $20 \mathrm{mM}$ and incubated for another hour with gentle rocking at $37^{\circ} \mathrm{C}$ followed by centrifugation at $10,000 \mathrm{~g}$ for 10 minutes. The supernatant was kept and samples were cleaned and desalted via solid phase extraction with Sep-Pak Plus cartridges (Waters Limited, Mississauga, Ontario), concentrated, solvent exchanged, filtered, aliquoted and frozen at $-80^{\circ} \mathrm{C}[14]$.

\section{D-LC MS/MS}

Each extracted and digested sample was analyzed twice for technical replicate in 2D-LC MS/MS. Samples were loaded onto the back column of split phase 2D column [15] $(\sim 3-5 \mathrm{~cm}$ Luna SCX $5 \mu \mathrm{m}$ and $\sim 3-5 \mathrm{~cm}$ $\mathrm{C}_{18}$ Aqua $5 \mu \mathrm{m} 100 \mathrm{~A}$ ) (Phenomenex, Torrance, CA) using a pressure cell and then connected to a front column with integrated nanospray tip ( $15 \mathrm{~cm}$ of Aqua $C_{18}$ Aqua $5 \mu \mathrm{m} \mathrm{100A}$, Phenomenex, Torrance, CA; $150 \mu \mathrm{m}$ with $15 \mu \mathrm{m}$ tip, New Objective, Woburn, MA). Samples were analyzed via 2-D LC-nanoESI-MS/MS on a LTQ XL (ThermoFisher Scientific, San Jose, CA) as previously described [13]. Samples were analyzed via 13-hr runs (6 steps with 1 wash step) $[14,16]$.

\section{Proteome informatics}

Proteome Discoverer 1.1 (ThermoFisher Scientific, San Jose, CA) and SEQUEST algorithm [17] was used to correlate peptides spectra with S. stipitis protein sequence (filtered models containing 5839 entries downloaded from the Joint Genome Institute; accessed on 05/15/2011) and $S$. cerevisiae protein sequence (5884 entries downloaded from the Saccharomyces Genome Database; accessed on 05/15/2011) [6,16]. Proteome Discoverer 1.1 was used to filter SEQUEST results and sort peptides into protein identification based on modified method of Verberkmoes et al. [17] (minimum Xcor of at least $1.5[+1], 2.3[+2]$, $2.8[+3]$; minimum probability score of at least $2[+1], 7[+2]$ and 7.5 $[+3]$; minimum two fully tryptic peptides) [13]. We enabled protein grouping, counted only rank 1 peptides and only in the top scored proteins. Each protein identified had at least one unique peptide. Reverse protein sequences were included in the search database to estimate the overall false-positive rates of protein identification (falsepositive rate $=2\left[\mathrm{n}_{\mathrm{rev}} /\left(\mathrm{n}_{\mathrm{rev}}+\mathrm{n}_{\mathrm{real}}\right)\right]^{\star} 100 ; \mathrm{n}_{\mathrm{rev}}=$ the number of peptides identified from the reverse database; $n_{\text {real }}^{\text {rev }}=$ the number of peptides identified from the real database) [18].

\section{Data analysis}

KEGG and GO annotations of S. cerevisiae and S. stipitis (also KOGs) were downloaded from Saccharomyces Genome Database and Joint Genome Institute $[19,20]$. The identified proteomes were examined manually with each biological function. In-house Perl scripts were written to perform the functional counts of GOs and KOGs. The metabolic pathways were visualized using KEGG Mapper [21]. For comparison of protein abundance levels, normalized spectral abundance factors (as label free estimation of relative protein abundance) (NSAF) were determined as described in Florens et al. [22]. This method estimates protein abundance by first dividing the spectral count for each protein by the protein length and then dividing this number by the sum of all length-normalized spectral counts for each organism and multiplying by 100 [22,17]. Differentially expressed proteins between 11.5 hour and 32-hour were determined using spectral count and the software QSpec with log. fold change of 3 and up-regulated FDR $=0.01$ [23].

\section{Results and Discussions}

\section{Overview of proteome}

This study represented the first attempt to monitor the temporal physiological profile of a co-culture process using S. cerevisiae and S. stipitis with shotgun proteomics. Key metabolites, including ethanol, glucose, acetic acid and glycerol were measured and monitored throughout the co-culture fermentation (Figure 1 and 2). The fermentation lasted for 36 hour in both biological replicates. The highest ethanol yielded $\sim 9 \mathrm{~g} / \mathrm{L}$. We observed low acetic acid $(<0.5 \mathrm{~g} / \mathrm{L})$ throughout the process, but glycerol was observed at moderate level $(<1.15 \mathrm{~g} / \mathrm{L})$. Glucose was consumed to near depletion $(<1 \mathrm{~g} / \mathrm{L})$ at $\sim 16$ hour, indicating diauxic shift for both organisms. We inoculated batch co-culture fermentation with the pre-inoculums and obtained initial cell density of $8.07 \times 10^{5} \mathrm{CFU}$ for $S$. cerevisiae and $7.23 \times 10^{5} \mathrm{CFU}$ for $S$. stipitis in biological replicate 1 at time zero. In biological replicate 2 we obtained initial cell density of $7.87 \times 10^{5} \mathrm{CFU}$ for S. cerevisiae and 1.10 $\mathrm{x} 10^{6} \mathrm{CFU}$ for $S$. stipitis. Before 11.5 hour, both $S$. cerevisiae and S. stipitis multiplied at near equal rate. In biological replicate $1, S$. stipitis outgrew S. cerevisiae, reaching $1.06 \times 10^{8} \mathrm{CFU}$ for $S$. stipitis and $6.20 \times 10^{7} \mathrm{CFU}$ for $S$. cerevisiae at the end of the fermentation. However, in biological replicate 2, the population of $S$. stipitis and S. cerevisiae fluctuated back and forth, failing to determine the dominant specie. This study further demonstrated the challenge in producing consistent batches in co-culture process. Based on the growth curve of each organism, the maximal growth rates of both S. cerevisiae and S. stipitis occurred at 11.5 hour and the maximal glucose consumption rate was also observed 
Citation: Huang EL, Lefsrud MG (2014) Fermentation Monitoring of a Co-Culture Process with Saccharomyces cerevisiae and Scheffersomyces stipitis Using Shotgun Proteomics. J Bioprocess Biotech 4: 144 doi: 10.4172/2155-9821.1000144

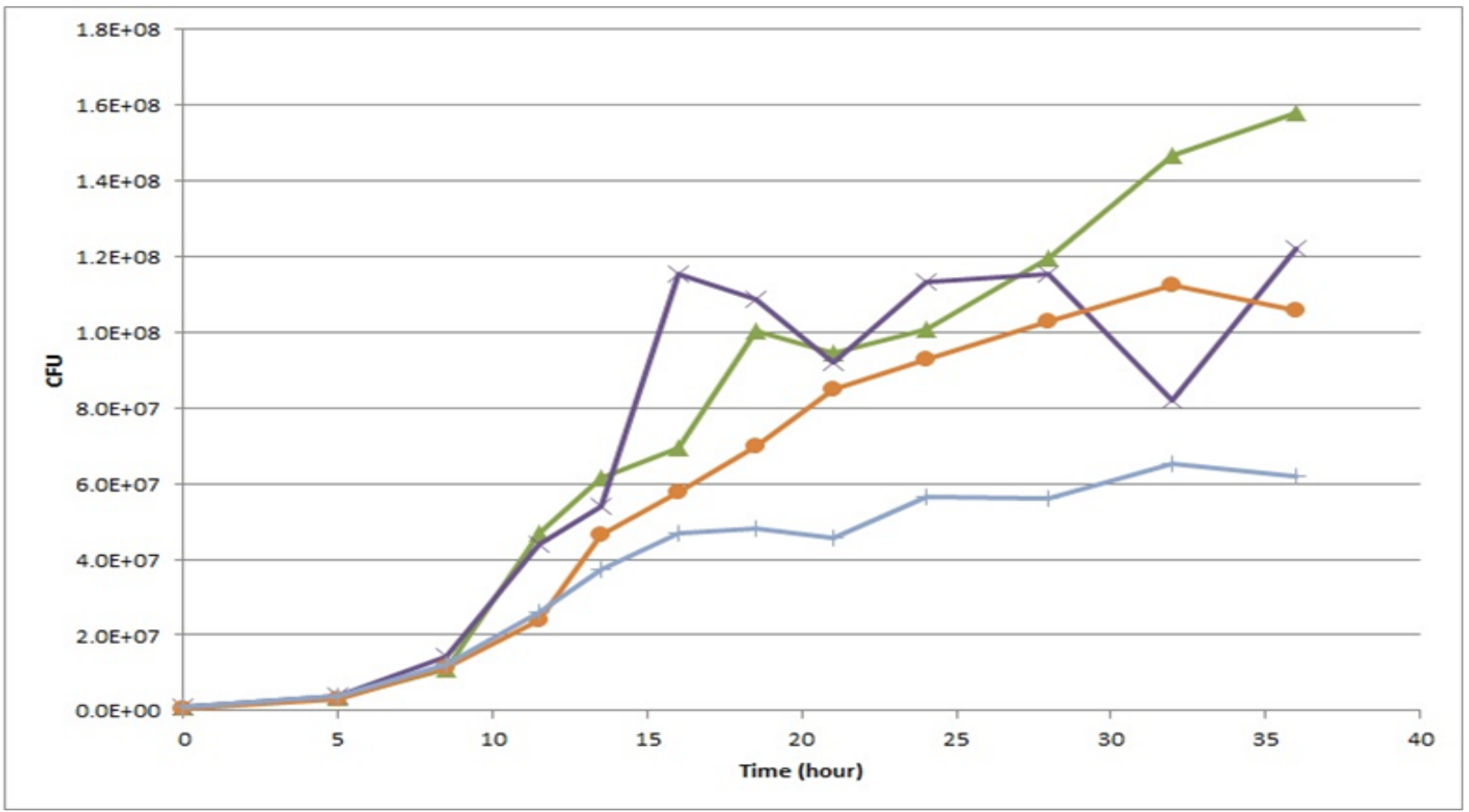

Figure 1: Viable cell counts of the co-culture process of Saccharomyces cerevisiae and Scheffersomyces stipitis in both biological replicate 1 and 2. Proteomics samples were taken at 11.5 hour, 18.5 hour, 32 hour, representing transition into diauxic shift. Fermentation was terminated at 36 -hour (•S. stipitis CFUs Biological 1; $\boldsymbol{\Delta}$ S. stipitis CFUs Biological 2; + S. cerevisiae CFUs Biological 1; X S. cerevisiae CFUs Biological replicate 2).

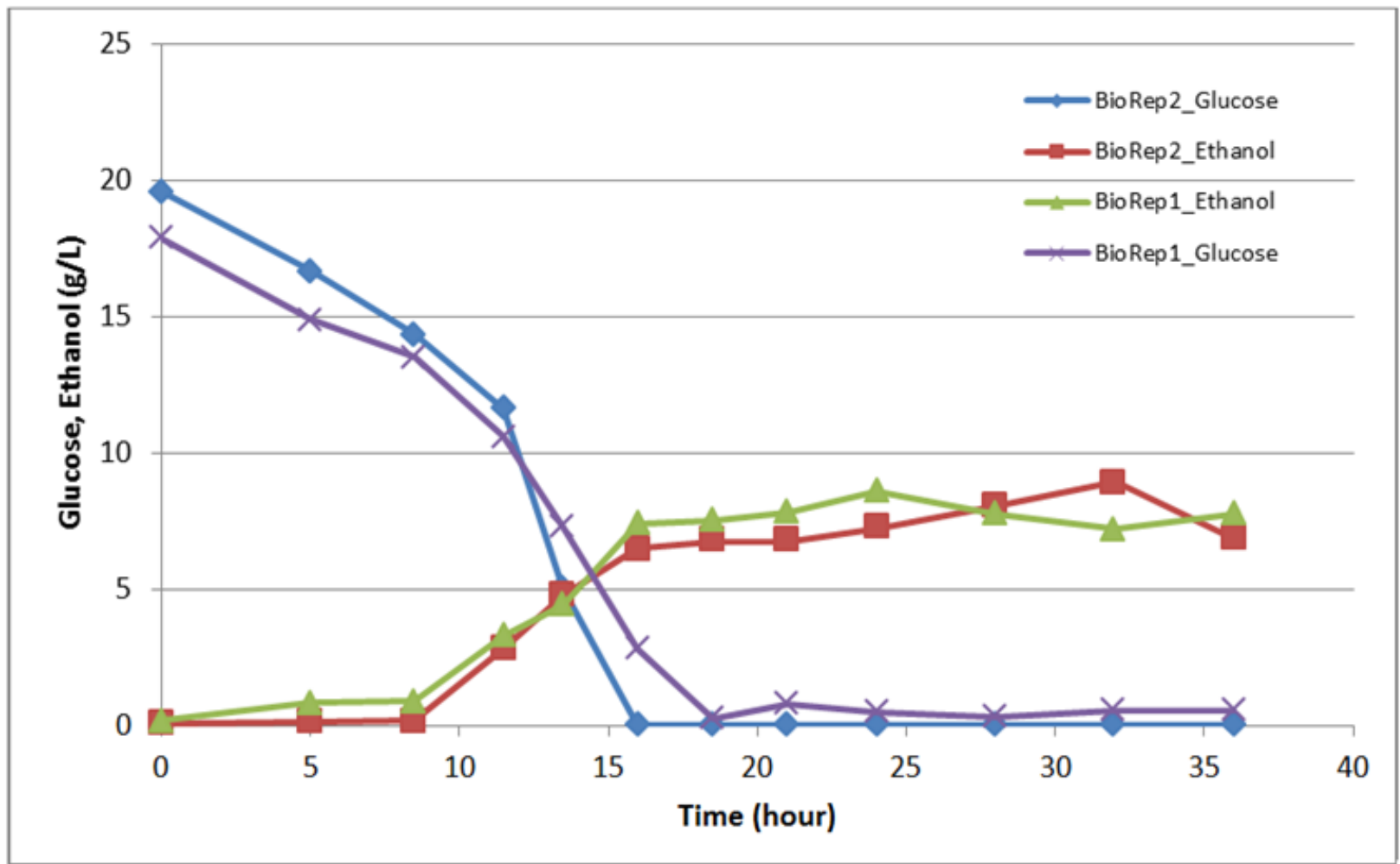

Figure 2: Monitoring glucose and ethanol concentrations during the co-culture process of Saccharomyces cerevisiae and Scheffersomyces stipitis. Data shown included both biological replicate 1 and 2 . 
at 11.5 hour, corresponding to the exponential growth phase of both yeasts. We collected proteomics samples from two flask fermentations at three time points (11.5 hour, 18.5 hour and 32 hour) to monitor the co-culture process. The shotgun approach was used to identified and matched peptide mass data to the sequenced S. stipitis and $S$. cerevisiae sequence databases. In biological replicate 1 , combining all time points and technical replicates for each time point, we identified a total number of 1390 proteins throughout the co-culture process (650 proteins from S. stipitis and 740 proteins from S. cerevisiae (Table 1). In biological replicate 2 , combining all time points and technical replicates for each time point, we identified a total number of 1499 proteins throughout the co-culture process ( 666 proteins from S. stipitis and 833 proteins from $S$. cerevisiae; Table 1). The false discovery rates of $2 \mathrm{D}$-LC-MS/MS runs were calculated between $0.16 \%$ to $0.57 \%$. Table 1 summarized spectral counts and the CFU for each organism in each time point in both biological replicates. We observed that although both $S$. cerevisiae and $S$. stipitis CFU were at approximately $1: 1$ ratio $\left(\sim 2.5 \times 10^{7} \mathrm{CFU}\right.$ each $)$ at 11.5 hour, the total peptides counts were not equal between two yeasts. The difference between total peptides counts demonstrated the difference in cell size and mass between S. stipitis and S. cerevisiae, where S. stipitis is smaller than S. cerevisiae in cell mass per cell. S. stipitis exhibited higher CFU than S. cerevisiae during the fermentation, but higher spectral counts in S. cerevisiae were observed. Looking at the spectral counts, CFU and protein identified, we found an increase in $S$. cerevisiae CFU did not necessary result in identifications of higher proteins and spectral counts, but an increase in S. stipitis CFU resulted in higher spectral counts and minor increase in numbers of protein identification. This implied other variables, which could be time dependent, interacted with spectrum identification capacity of the mass spectrometry. One possible explanation could be the competition for electro-spray ionization between S. cerevisiae and S. stipitis peptides. Another explanation was the difference in extraction and digestion efficiency between two organisms. We calculated normalized spectral abundance factors (NSAFs) to estimate the relative protein abundance. By comparing the technical replicates of each dataset, we obtained highly reproducible results in each biological replicate, with the linear regression line fell between $\mathrm{R}^{2}=0.81$ and 0.89 for $S$. cerevisiae in biological replicate 1 (as illustrated in Figure 3 for 18.5 hour time point) and between $\mathrm{R}^{2}=0.85$ and 0.90 for S. stipitis (data not shown).

\section{Most abundant proteins in s. cerevisiae and s. stipitis}

Proteomics data was further analyzed below by using both biological replicates if not stated. Similar to the most abundant proteins found using GFP fusion library and high-throughput flow cytometry, S. cerevisiae glycolytic enzymes were among the most abundant proteins in the cell from exponential to diauxic shift [24]. These included pyruvate kinase (Cdc19p), 3-phosphoglycerate kinase (Pgk1p), glyceraldehyde-3-phosphate dehydrogenase (Tdh1p,
Tdh2p, Tdh3p), enolase II (Eno2p) and fructose 1,6-bisphosphate aldolase (Fba1p). Alcohol dehydrogenase (Adh1p) and F1 sector of mitochondrial F1F0 ATP synthase (Atp2p) were also highly abundant in $S$. cerevisiae proteome, indicating the importance of oxidative phosphorylation. The fact that the other enzymes of the tricarboxylic acid cycle were not switched off during fermentative growth can be explained by their involvement in amino acid biosynthesis, gluconeogenesis, heme biosynthesis or de novo fatty acid synthesis.

The most abundant proteins identified in S. stipitis also came from glycolysis and gluconeogenesis. Similar to $S$. cerevisiae, the glycolytic enzymes observed in most abundance included glyceraldehyde-3phosphate dehydrogenase (Tdh2p and Tdh3p), enolase I (Eno1p), phosphoglycerate mutase (Gpm1.1p), fructose-bisphosphate aldolase (Fbalp), phosphoglycerate kinase (Pgk13p) and pyruvate kinase (Pyk1p). S. stipitis cells also produced high amount of alcohol dehydrogenase (Adh1p), which reduces acetaldehyde to ethanol. We observed high abundance of $\mathrm{F}_{1} \mathrm{~F}_{0}$-ATPase complex alpha and beta subunits (Atp1p, Atp2p) located in mitochondrial cellular components. We also identified high abundance of mitochondrial malate dehydrogenase (Mdhlp) involved in tricarboxylic acid (TCA) cycle, indicating respiro-fermentative. Proteins involved in chromatin assembly, protein translation and folding were also high in abundance. These included heat shock protein (HSP70 family Ssa2p), histone $\mathrm{H} 4$ and 2B (Hhf1p and Htb2.1p) and ribosomal proteins (L14B, 40S S14-B, S7-A and 60S L4360S). The resemblance in both S. cerevisiae and S. stipitis proteomes showed that highly abundant proteins were housekeeping proteins and essential for viability. In yeast cells, glycolytic enzymes constitute a major fraction (30 to 60\%) of the soluble proteins [25], and in rapidly growing cells, $60 \%$ of total transcription is devoted to ribosomal RNA [26].

\section{Functional categorization and analysis of s. cerevisiae and s. stipitis proteome}

A comparison between S. stipitis and other more related yeast such as C. albicans would almost certainly show a higher degree of conservation, but because regulation has been best studied in $S$. cerevisiae, that is a standard frame of reference. Therefore in this study regulation of $S$. stipitis was inferred and compared with $S$. cerevisiae if not otherwise stated. The proteome of S. cerevisiae and S. stipitis observed in the co-cultural process contained housekeeping processes such as oxidative phosphorylation, glycolysis, non-oxidative branch of the pentose phosphate pathway, gluconeogenesis, biosynthesis of amino acids and aminoacyl-tRNA, protein synthesis and proteolysis, fatty acid metabolism and cell division. An attempt was made to categorize $S$. stipitis proteome based on GO terms for direct comparison between S. stipitis and S. cerevisiae, but the incomplete nature of the GO entries for S. stipitis proved impractical (data not shown). The incomplete

\begin{tabular}{|c|c|c|c|c|}
\hline & \multicolumn{3}{|c|}{ Number of Proteins Identified Biological Replicate 1} & \multirow[t]{2}{*}{ Total proteins measured from each organism } \\
\hline & 11.5 hour & 18.5 hour & 32 hour & \\
\hline Scheffersomyces stipitis & 322 & 456 & 574 & 650 \\
\hline Saccharomyces cerevisiae & 535 & 594 & 539 & 740 \\
\hline \multirow[t]{3}{*}{ Total proteins in each time-point } & 857 & 1050 & 1113 & 1390 \\
\hline & \multicolumn{3}{|c|}{ Number of Proteins Identified Biological Replicate 2} & Total proteins measured from each organism \\
\hline & 11.5 hour & 18.5 hour & 32 hour & \\
\hline Scheffersomyces stipitis & 444 & 412 & 577 & 666 \\
\hline Saccharomyces cerevisiae & 626 & 637 & 660 & 833 \\
\hline Total proteins in each time-point & 857 & 1050 & 1113 & 1499 \\
\hline
\end{tabular}

Table 1: Numbers of Scheffersomyces stipitis and Saccharomyces cerevisiae proteins identified from shotgun proteomics of the co-culture process in both biological replicates. 
Citation: Huang EL, Lefsrud MG (2014) Fermentation Monitoring of a Co-Culture Process with Saccharomyces cerevisiae and Scheffersomyces stipitis Using Shotgun Proteomics. J Bioprocess Biotech 4: 144 doi: 10.4172/2155-9821.1000144

NSAF Bio1_T11.5a vs. Bio1_T11.5b

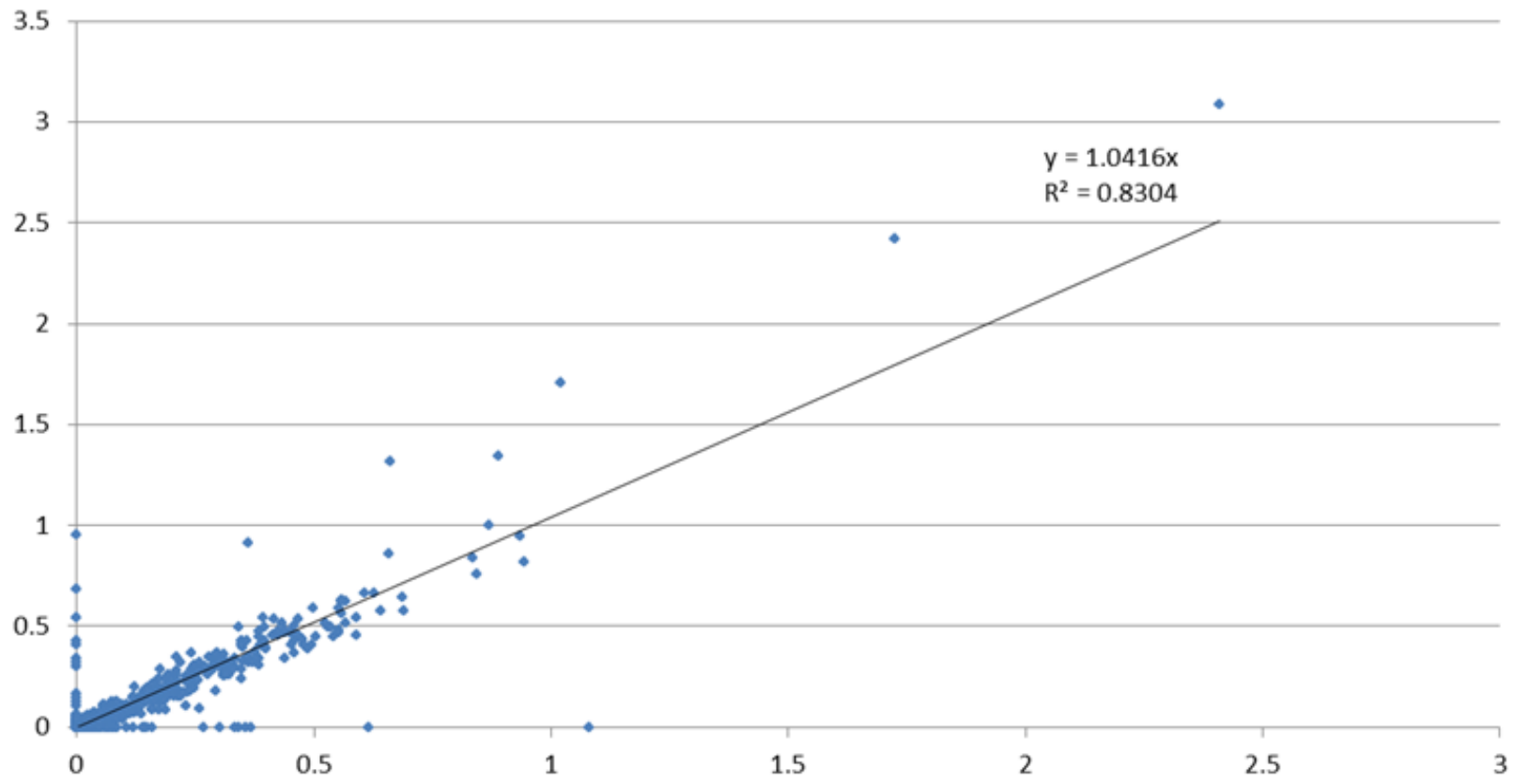

Figure 3: Representative linear regression based on NSAFs of S. cerevisiae using technical replicate a and technical replicate $b$ at 18.5 hour in biological replicate 1.

nature of the GO annotations for S. stipitis directed us to categorize $S$. stipitis proteome based on KOGs. In analyzing the $S$. stipitis proteome, the majority of KOGs functions fell into the categories of carbohydrate metabolism, protein synthesis and modification. Technical replicates of each time point showed reproducible counts of each KOGs term. Even with the higher counts observed in later time points, each KOGs category still constituted the same relative ratio to the whole data set (data now shown). The majority of GO terms also fell into protein synthesis and carbohydrate metabolism in S. cerevisiae. The ratio of each category relative to the whole data set was consistent between time points. Technical replicate of each time point also showed reproducibility. All time points shared similar distribution of biological function in terms of KOGs for S. stipitis and GO Biological terms for S. cerevisiae.

\section{Distinctive physiology in temporal proteome}

In analyzing the proteome of $S$. stipitis, certain stress proteins were observed before diauxic shift, and these included thioredoxin peroxidase (acts as antioxidant) and glycogen synthase (Gsylp). The expression of Gsylp was observed as early as 11.5 hour in both S. cerevisiae and S. stipitis. It was possible that heat shock, nitrogen starvation or the $2 \%$ glucose medium (insufficient glucose) caused the de-repression of Gsylp [27,28]. Earlier studies suggested S. cerevisiae and S. stipitis shared similar amino acid biosynthesis pathway. In S. stipitis proteome, we observed proteins involved in a list of amino acid biosynthesis pathways that are under general amino acid controls before diauxic shift. These included acetolactate synthase (Ilv2p), dihydroxyacid dehydratase (Ilv3p, 32 hour only), mitochondrial ketolacid reductoisomerase (Ilv5p) and 3-deoxy-D-arabino-heptulosonate 7-phosphate synthase isoenzyme (Aro4p). Hans and colleagues observed the most drastic alternations of intracellular amino acid pools occurred during the diauxic shift and at the entry into the stationary phase [29]. We also observed principal enzyme of glyoxylate cycle before diauxic shift, including malate synthase (Mls1.1p) and isocitrate lyase (Icl1p), a key enzyme for growth on ethanol and acetate in yeast. Earlier studies in S. cerevisiae showed that ICL1 expression is tightly regulated and growth on ethanol requires the glyoxylate pathway for replenishing C4 compounds to the tricarboxylic acid cycle. ICL1 was also induced by growth on ethanol and repressed by growth on glucose [30]. The most peculiar finding in our co-culture process was the observation of the enzymes involved in oxo-reductive pathway for xylose utilization. We observed $\mathrm{NAD}(\mathrm{P}) \mathrm{H}$-dependent $\mathrm{D}$-xylose reductase (Xyllp) as early as 11.5- hour before glucose depletion. We also observed D-xylulose reductase as early as 18.5-hour after glucose depletion. Xylose was not presented in the media (confirmed by enzymatic assay). We observed aldehyde dehydrogenase (Ald5p) at 32 hour, but S. stipitis was not known to produce acetate. The function of Ald5p remained to be examined. Our metabolic data supported the expression of DLglycerol-3-phosphatase (Gpplp) and suggested anaerobic and osmotic stress for S. stipitis. Other proteins which suggested stress included Gph1p, glycogen (starch) synthase (Gsylp), thiol-specific antioxidant protein (Tsa1p), NADPH-dependent methylglyoxal reductase (Gre2p) and thioredoxin reductase (Prxlp) [31,32].

\section{Dynamic of S. stipitis during fermentation}

Using the spectral counts and the software QSpec (log. fold change cutoff $=3$; FDR up-regulated $=0.01$ ) [23], we identified up-regulations of six $S$. stipitis proteins and one S. cerevisiae protein from 11.5 hour to 32 hour (Table 2). During the diauxic shift at 32-hour, we observed up-regulation of $S$. stipitis malate synthase and isocitrate lyase, the key enzymes involved in glyoxylate cycle. The depletion of glucose and the transition to growth on ethanol and acetate also triggered the up-regulation of $S$. stipitis phosphoenolpyruvate carboxykinase in gluconeogenesis. Interestingly, S. stipitis histone 2B did not exhibit 
Citation: Huang EL, Lefsrud MG (2014) Fermentation Monitoring of a Co-Culture Process with Saccharomyces cerevisiae and Scheffersomyces stipitis Using Shotgun Proteomics. J Bioprocess Biotech 4: 144 doi: 10.4172/2155-9821.1000144

Page 6 of 7

\begin{tabular}{|c|c|c|c|c|c|c|c|c|c|}
\hline Organism Accession & Bio1 11.5hr & Bio2 11.5hr & Bio1 32hr & Bio2 32hr & Fold Change (log) & Z statistics & FDR & FDR Up-regulated & Description \\
\hline S. stipitis 89873 & 0 & 0 & 41 & 26 & 3.185 & 4.94 & 0.021 & 0.006 & Acetyl-coenzyme A synthetase \\
\hline 66312 & 0 & 0 & 10 & 40 & 3.228 & 5.28 & 0.01 & 0.002 & Endo-1,4-beta-glucanase \\
\hline 73488 & 0 & 0 & 49 & 32 & 3.465 & 5 & 0.018 & 0.005 & 0 \\
\hline 53620 & 0 & 0 & 45 & 64 & 3.78 & 6.27 & 0.001 & Histone 2B & 0 \\
\hline 62080 & 0 & 0 & 96 & 88 & 4.411 & 6.47 & 0 & Malate synthase, glyoxysomal \\
\hline 71471 & 0 & 0 & 58 & 123 & 4.941 & 5.32 & 0.009 & 0.002 \\
\hline S. cerevisiae YBL075c & 0 & 0 & 0 & 122 & 3.989 & 5.95 & 0.002 & Phosphoenolpyruvate car- \\
\hline
\end{tabular}

Table 2: Up-regulated proteins of S. stipitis and S. cerevisiae from 11.5 hour to 32 hour. Differentially expressed proteins were determined using spectral counts and the software QSpec with the cutoff of 3 log fold change and FDR Up-regulated of 0.01

down-regulation and increased histone acetylation was suggested by up-regulation of acetyl-coA synthetase. With glucose repression no longer existed at 32 hour, we also observed up-regulation of $S$. stipitis endo-1,4-beta-glucanase, a family 5 glycoside hydrolase, without cellulose in the growth media. Another unexpected finding in our proteomics data was the observation of the expressed enzymes involved in oxo-reductive pathway of xylose utilization in S. stipitis. $\mathrm{NAD}(\mathrm{P}) \mathrm{H}$-dependent $\mathrm{D}$-xylose reductase (Xyllp) and D-xylose reductase (Xyl2p) are repressed under glucose culture [33]. However, we observed S. stipitis Xyllp expression as early as 11.5- hour before glucose depletion. We also observed S. stipitis Xyl2p expression as early as 18.5 hour after glucose depletion. Xylose was not present in the media (confirmed by enzymatic assay) and the mechanism for signal derepression of these two enzymes is unknown. Earlier studies have suggested that reducing ATP production can also bring about a partial derepression of xylose assimilation [34]. Further study is needed to investigate and verify the derepression signal for Xyllp and Xyl2p. We hypothesize that the derepression signal came from the effect of co-culture, which could be a competitive advantage for S. stipitis [35] as a hedging bet to utilize other potential carbon sources. Along with the up-regulation of S. stipitis endo-1,4-beta-glucanase without cellulose induction, our data demonstrated S. stipites strategy in diversifying potential carbon sources once glucose no longer existed.

\section{Conclusions}

In this study, we demonstrated a shotgun proteomics to monitor a co-culture processing with S. cerevisiae and S. stipitis. Shotgun proteomics successfully distinguished, identified distinct physiology of both yeast during the co-culture process, and able to elucidate the temporal profile of $S$. stipitis proteome. The most abundant proteins of $S$. stipitis and S. cerevisiae came from the expected process such as glycolytic enzymes, histones, heat shock proteins, ribosomal proteins and $\mathrm{F}_{1} \mathrm{~F}_{0}$-ATPase. Without the presence of xylose, the induction of $\mathrm{NAD}(\mathrm{P}) \mathrm{H}$-dependent $\mathrm{D}$-xylose reductase and $\mathrm{D}$-xylulose reductase were unexpected. Up-regulation of S. stipitis endo-1,4-beta-glucanase, histone $2 \mathrm{~B}$ and acetyl-coA synthetase provided insight into the dynamics of non-model yeast $S$. stipitis during the co-culture process.

\section{Acknowledgments}

We thank Brian Dill and Collin Olivier for giving us detailed parameters of datadepended and LTQ XL setup. Funding of the project came from McGill University start up fund and Canadian Foundation for Innovation (CFI-IOF grant \#23635)

\section{References}

1. Kleerebezem R, Van Loosdrecht MCM (2007) Mixed Culture Biotechnology for Bioenergy Production. Curr Opin Biotechnol 18: 207-212.

2. Florens L, Carozza MJ, Swanson SK, Fournier M, Coleman MK, et.al., (2006) Analyzing Chromatin Remodeling Complexes using Shotgun Proteomics and Normalized Spectral Abundance Factors. Methods 40: 303-311.

3. Reis MAM, Serafim LS, Lemos PC, Ramos AM, Aguiar FR, et.al., (2003)
Production of Polyhydroxyalkanoates by Mixed Microbial Cultures. Bioprocess Biosyst Eng 25: 377-385.

4. Rodrguez J, Kleerebezem R, Lema JM, Van Loosdrecht M (2006) Modeling Product Formation in Anaerobic Mixed Culture Fermentations. Biotechnol Bioeng 93: 592-606.

5. Ciani M, Comitini F, Mannazzu I, Domizio P (2010) Controlled Mixed Culture Fermentation: A New Perspective on the use of Non-Saccharomyces Yeasts in Winemaking. FEMS Yeast Res 10: 123-133.

6. Awafo VA, Chahal DS, Simpson BK (1998) Optimization of Ethanol Production by Saccharomyces cerevisiae (ATCC 60868) and Pichia stipitis Y-7124: A Response Surface Model for Simultaneous Hydrolysis and Fermentation of Wheat Straw. J Food Biochemistry 22: 489-509.

7. Taniguchi M, Itaya T, Tohma T, Fujii M (1997) Ethanol Production from a Mixture of Glucose and Xylose By a Novel Co-Culture System with Two Fermentors and Two Microfiltration Modules. J Biosci Bioeng 84: 59-64.

8. Milburn M (2009) Using Metabolic Profiling Technology to Advance Cell Culture Development. Biopharm International Supplements.

9. Pham TK, Chong PK, Gan CS, Wright PC (2006) Proteomic Analysis of Saccharomyces Cerevisiae under High Gravity Fermentation Conditions. J Proteome Res 5: 3411-3419.

10. Washburn MP, Wolters D, Yates III JR (2001) Large-Scale Analysis of the Yeast Proteome by Multidimensional Protein Identification Technology. Nat Biotechnol 19: 242-247.

11. Laplace JM, Delgenes JP, Moletta R, Navarro JM (1992) Alcoholic Glucose and Xylose Fermentations by the Coculture Process: Compatibility and Typing of Associated Strains. Can J Microbiol 38: 654-658.

12. Thompson MR, Chourey K, Froelich JM, Erickson BK, VerBerkmoes NC (2008) Experimental Approach for Deep Proteome Measurements from Small-Scale Microbial Biomass Samples. Anal Chem 80: 9517-9525.

13. Wilmes P, Andersson AF, Lefsrud MG, Wexler M, Shah M, et.al., (2008) Community Proteogenomics Highlights Microbial Strain-Variant Protein Expression within Activated Sludge Performing Enhanced Biological Phosphorus Removal. The ISME J 2:853-864

14. McDonald WH, Ohi R, Miyamoto DT, Mitchison TJ, Yates JR (2002) Comparison of Three Directly Coupled HPLC MS/MS Strategies for Identification of Proteins from Complex Mixtures: Single-Dimension LC-MS/MS, 2-Phase Mudpit, And 3-Phase Mudpit. Int J Mass 219: 245-251.

15. Eng JK, McCormack AL, Yates JR (1994) An Approach to Correlate Tandem Mass Spectra Data of Peptides with Amino Acid Sequences in a Protein Database. J Am Soc Mass Spectrom 5: 976-989.

16. Goltsman DSA, Denef VJ, Singer SW, VerBerkmoes NC, Lefsrud M, et.al. (2009) Community Genomic And Proteomic Analyses of Chemoautotrophic Iron-Oxidizing" Leptospirillum Rubarum"(Group II) and "Leptospirillum Ferrodiazotrophum"(Group III) Bacteria in Acid Mine Drainage Biofilms. App Environ Microbiol 75: 4599-4615.

17. Verberkmoes NC, Russell AL, Shah M, Godzik A, Rosenquist M, et.al., (2008) Shotgun Metaproteomics of the Human Distal Gut Microbiota. The ISME J 3:179-189.

18. Cherry JM, Adler C, Ball C, Chervitz SA, Dwight SS, et al. (1998) SGD: Saccharomyces Genome Database. Nucl Acids Res 26: 73-79.

19. Jeffries TW, Grigoriev IV, Grimwood J, Laplaza JM, Aerts A, et.al., (2007) Genome Sequence of the Lignocellulose-Bioconverting and Xylose-Fermenting Yeast Pichia stipitis. Nat Biotechnol 25: 319-326. 
Citation: Huang EL, Lefsrud MG (2014) Fermentation Monitoring of a Co-Culture Process with Saccharomyces cerevisiae and Scheffersomyces stipitis Using Shotgun Proteomics. J Bioprocess Biotech 4: 144 doi: 10.4172/2155-9821.1000144

20. Kanehis, M, Goto S (2000) KEGG: Kyoto encyclopedia of Genes and Genomes. Nucleic Acids Res 28: 27-30.

21. Choi H, Fermin D, Nesvizhskii Al (2008) Significance Analysis of Spectral Coun Data in Label-free Shotgun Proteomics. Mol Cell Proteomics 7: 2373-2385.

22. Davidson GS, Joe RM, Roy S, Meirelles O, Allen CP, et.al., (2011) The proteomics of quiescent and nonquiescent cell differentiation in yeast stationary-phase cultures. MBoC 22: 988-998.

23. Fernandez E, Moreno F, Rodicio R (1992) The ICL1 Gene from Saccharomyces cerevisiae. Eur J Biochem 204: 983-990.

24. Fu N, Peiris P, Markham J, Bavor J (2009) A Novel Co-Culture Process with Zymomonas mobilis and Pichia stipitis for Efficient Ethanol Production on Glucose/Xylose Mixtures. Enzyme Microb Tech 45: 210-217.

25. Hans MA, Heinzle E, Wittmann C (2001) Quantification of intracellular amino acids in batch cultures of Saccharomyces cerevisiae. Appl Microbiol Biotechnol 56: $776-779$.

26. Hwang PK, Tugendreich S, Fletterick RJ (1989) Molecular analysis of GPH1, the gene encoding glycogen phosphorylase in Saccharomyces cerevisiae. Mol Cell Biol 9: 1659-1666.

27. Unnikrishnan I, Miller S, Meinke M, LaPorte DC (2003) Multiple positive and negative elements involved in the regulation of expression of GSY1 in Saccharomyces cerevisiae. J Biol Chem 278: 26450-26457.

28. Pahlman AK, Granath K, Ansell R, Hohmann, S, Adler L (2001) The yeast glycerol 3-phosphatases Gpp1p and Gpp2p are required for glycerol biosynthesis and differentially involved in the cellular responses to osmotic anaerobic, and oxidative stress. J Biol Chem 276: 3555-3563.

29. Parrou JL, Enjalbert B, Plourde L, Bauche A, Gonzalez B, François J (1999) Dynamic responses of reserve carbohydrate metabolism under carbon and nitrogen limitations in Saccharomyces cerevisiae. Yeast 15: 191-203.

30. Peng J, Elias JE, Thoreen CC, Licklider LJ, Gygi SP (2003) Evaluation of multidimensional chromatography coupled with tandem mass spectrometry (LC/LC- MS/MS) for large-scale protein analysis: the yeast proteome. J Proteome Res 2: 43-50.

31. Scott EW, Baker HV (1993) Concerted Action of the Transcriptional Activators REB1, RAP1 and GCR1 in the High-level Expression of the Glycolytic Gene TPI. Mol Cell Biol 13: 543-550.

32. Sunnarborg SW, Miller SP, Unnikrishnan I, LaPorte DC (2001) Expression of the yeast glycogen phosphorylase gene is regulated by stress, Äêresponse elements and by the HOG MAP kinase pathway. Yeast 18: 1505-1514.

33. Shi NQ, Davis B, Sherman F, Cruz J, Jeffries TW (1999) Disruption of the cytochrome c gene in xylose-utilizing yeast Pichia stipitis leads to higher ethanol production. Yeast 15: 1021-1030.

34. Slininger PJ, Thompson SR, Weber S, Liu ZL, Moon J (2011) Repression of Xylose-Specific Enzymes by Ethanol in Scheffersomyces (Pichia) stipitis and Utility of Repitching Xylose-Grown Populations to Eliminate Diauxic Lag. Biotechnol Bioeng 108: 1801-1815.

35. Warner JR (2001) The Economics of Ribosome Biosynthesis in Yeast. Cold Spring Harb Symp Quant Biol 66: 567-574 